\title{
High-Power Terahertz Generation from Telecommunication-Compatible, Bias-Free Photoconductive Nano-Antennas
}

\author{
Deniz Turan ${ }^{1}$, Nezih T. Yardimci ${ }^{1}$, Zixuan Rong ${ }^{1}$, Dingkun Ren ${ }^{1}$, Hyunseok Kim ${ }^{1}$, Diana Huffaker ${ }^{1,2}$, and Mona Jarrahi ${ }^{1}$ \\ ${ }^{1}$ University of California, Los Angeles, Los Angeles, CA, 90095 USA \\ ${ }^{2}$ Cardiff University, Cardiff, Wales CF24 3AA, UK
}

\begin{abstract}
We present a telecommunication-compatible, biasfree photoconductive terahertz source with arrays of plasmonic nano-antennas. We demonstrate pulsed terahertz radiation with powers up to $72 \mu \mathrm{W}$, enabling time-domain terahertz spectroscopy over a $3 \mathrm{THz}$ bandwidth with a $100 \mathrm{~dB}$ dynamic range.
\end{abstract}

\section{INTRODUCTION}

$\mathrm{P}$ HOTOCONDUCTIVE terahertz sources are extensively used in time-domain terahertz spectroscopy (THz-TDS) systems for various imaging and sensing applications [1]-[5]. To generate terahertz pulses, a bias voltage is applied to a photoconductor illuminated by a femtosecond optical pump beam and the generated photocurrent is routed to a terahertz radiating element[6], [7]. It is highly desirable to develop photoconductive terahertz sources that can operate at telecommunication optical wavelengths $(\sim 1550 \mathrm{~nm})$, where low-cost, compact lasers are available[8]-[10]. However, photo-absorbing substrates at these optical wavelengths have low resistivity, which results in high dark currents and thermal breakdown before reaching high radiation powers.

To address this limitation and realize high-reliability, highperformance, telecommunication-compatible photoconductive terahertz source, we introduce a new type of photoconductive source, which can generate high terahertz radiation powers without a need for a bias voltage. The photoconductor is designed such that a built-in electric field induced inside the photo-absorbing substrate drifts the photo-generated carriers. As a result, relatively high photocurrent values feed the terahertz radiating elements at a zero dark current. We use arrays of plasmonic nano-antennas as the terahertz radiating elements, which provide broadband, high-power terahertz radiation [11]-[14].

\section{SOURCE DESIGN AND EXPERIMENTAL RESUlts}

The designed bias-free photoconductive terahertz source consists of a $1 \times 1 \mathrm{~mm}^{2}$ array of plasmonic nano-antennas fabricated on an InAs layer grown on a semi-insulating GaAs substrate. InAs is chosen as the photo-absorbing semiconductor because of its high carrier mobility. The InAs layer is highly pdoped $\left(1.4 \times 10^{19} \mathrm{~cm}^{-3}\right)$ to introduce a strong built-in electric field at the interface between InAs and the Ti/Au plasmonicnano-antennas (Fig. 1a). When the terahertz source is illuminated with a femtosecond optical excitation, the photogenerated electrons drift to the plasmonic nano-antennas by the built-in electric field and feed the nano-antennas with the ultrafast current required for generating terahertz pulses.

Geometry of the plasmonic nano-antennas (Ti/Au gratings with a $450 \mathrm{~nm}$ periodicity, $370 \mathrm{~nm}$ metal width, and $80 \mathrm{~nm}$ metal height, coated with a $240 \mathrm{~nm}$-thick $\mathrm{Si}_{3} \mathrm{~N}_{4}$ anti-reflection coating) is chosen to maximize optical absorption at the InAs$\mathrm{Ti} / \mathrm{Au}$ interface. Length of the nano-antennas $(5 \mu \mathrm{m})$ is chosen to be much smaller than terahertz wavelengths to maintain a broadband radiation. Optical and scanning electron microscope images of a fabricated terahertz source are shown in Fig. 1b. (a)

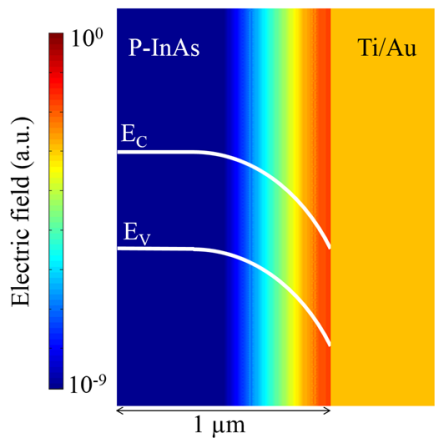

(b)

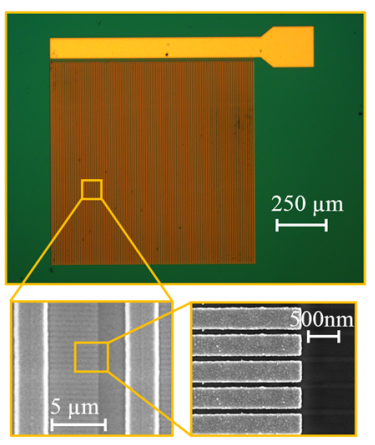

Fig. 1 (a) Band diagram (white lines) and built-in electric field (color plot) at the interface between the InAs substrate and the plasmonic nano-antennas. (b) Optical and scanning electron microscope images of the fabricated photoconductive terahertz source.

Performance of the fabricated terahertz source is characterized using an optical parametric oscillator that generates optical pulses with a $1550 \mathrm{~nm}$ center wavelength, 76 $\mathrm{MHz}$ repetition rate, and $100 \mathrm{fs}$ pulse width. The generated terahertz power is measured using a pyroelectric detector calibrated by Physikalisch-Technische Bundesanstalt (PTB), Germany. Power measurement results (Fig. 2a) show up to 72 $\mu \mathrm{W}$ radiated terahertz power from the fabricated terahertz source. Radiation spectrum of the terahertz source is analyzed using a THz-TDS system. The measured time-domain electric field (Fig. 2b) and the corresponding power spectrum (Fig. 2c) for the terahertz source indicate radiation bandwidths exceeding $3 \mathrm{THz}$ with a $100 \mathrm{~dB}$ dynamic range.
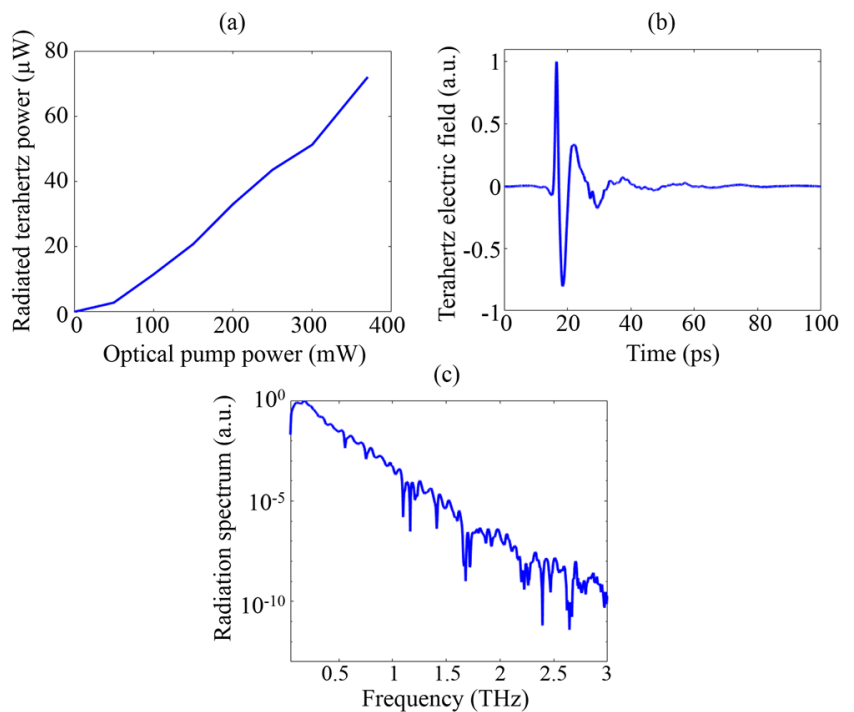

Fig. 2: The measured terahertz power, time-domain electric field, and radiation spectrum of the fabricated photoconductive terahertz source are shown in a, b, and $\mathrm{c}$, respectively. 


\section{CONCLUSION}

To summarize, a bias-free and telecommunicationcompatible photoconductive terahertz source, which offers radiation powers exceeding $72 \mu \mathrm{W}$ over a $3 \mathrm{THz}$ bandwidth, is introduced. The presented terahertz source would significantly

improve the performance, reliability, and practicality of THzTDS systems for various imaging and sensing applications.

\section{REFERENCES}

[1] B. B. Hu and M. C. Nuss, "Imaging with terahertz waves," Opt. Lett., vol. 20, no. 16, pp. 1716-1718, 1995.

[2] R. M. Woodward, V. P. Wallace, D. D. Arnone, E. H. Linfield, and M. Pepper, "Terahertz pulsed imaging of skin cancer in the time and frequency domain," J. Biol. Phys., vol. 29, no. 2-3, pp. 257-261, 2003.

[3] D. Grischkowsky, S. Keiding, M. van Exter, and C. Fattinger, "Farinfrared time-domain spectroscopy with terahertz beams of dielectrics and semiconductors," J. Opt. Soc. Am. B, vol. 7, no. 10, pp. 2006-2015, 1990.

[4] J. F. Federici et al., "THz imaging and sensing for security applications - explosives, weapons and drugs," Semicond. Sci. Technol., vol. 20, no. 7, pp. 266-280, 2005.

[5] T. Bowman et al., "Pulsed terahertz imaging of breast cancer in freshly excised murine tumors," J. Biomed. Opt., vol. 23, no. 02, p. 026004, 2018.

[6] P. U. Jepsen, R. H. Jacobsen, and S. R. Keiding, "Generation and detection of terahertz pulses from biased semiconductor antennas," $J$. Opt. Soc. Am. B, vol. 13, no. 11, pp. 2424-2436, 1996.

[7] D. H. Auston, K. P. Cheung, and P. R. Smith, "Picosecond photoconducting Hertzian dipoles," Appl. Phys. Lett., vol. 45, no. 3, pp. 284-286, 1984.

[8] S. Preu, M. Mittendorff, H. Lu, H. B. Weber, S. Winnerl, and A. C. Gossard, "1550 nm ErAs:In(Al)GaAs large area photoconductive emitters," Appl. Phys. Lett., vol. 101, no. 101105, 2012.

[9] N. Vieweg et al., "Terahertz-time domain spectrometer with $90 \mathrm{~dB}$ peak dynamic range," J. Infrared, Millimeter, Terahertz Waves, vol. 35, no. 10, pp. 823-832, 2014.

[10] B. Sartorius et al., "All-fiber terahertz time-domain spectrometer operating at $1.5 \mu \mathrm{m}$ telecom wavelengths," Opt. Express, vol. 16, no. 13, pp. 9565-9570, 2008.

[11] N. T. Yardimci, S. H. Yang, C. W. Berry, and M. Jarrahi, "High-power terahertz generation using large-area plasmonic photoconductive emitters," IEEE Trans. Terahertz Sci. Technol., vol. 5, no. 2, pp. 223229, 2015.

[12] N. T. Yardimci, H. Lu, and M. Jarrahi, "High power telecommunicationcompatible photoconductive terahertz emitters based on plasmonic nano-antenna arrays," Appl. Phys. Lett., vol. 109, no. 19, p. 191103, 2016.

[13] N. T. Yardimci, R. Salas, E. M. Krivoy, H. P. Nair, S. R. Bank, and M. Jarrahi, "Impact of substrate characteristics on performance of large area plasmonic photoconductive emitters," Opt. Express, vol. 23, no. 25, pp. 32035-32043, 2015.

[14] D. Turan, S. C. Corzo-Garcia, N. T. Yardimci, E. Castro-Camus, and M. Jarrahi, "Impact of the metal adhesion layer on the radiation power of plasmonic photoconductive terahertz sources," vol. 38, no. 12, pp. 14481456, 2017. 